\title{
Inducing Alcohol Dependence in Rats Using Chronic Intermittent Exposure to Alcohol Vapor
}

Elizabeth M Avegno ${ }^{1}$ and Nicholas W Gilpin ${ }^{1,2, *}$

\author{
${ }^{1}$ Department of Physiology, Louisiana State University Health Science Center, New Orleans, LA 70112, \\ USA; ${ }^{2}$ Neuroscience Center of Excellence, Louisiana State University Health Science Center, New \\ Orleans, LA 70112, USA \\ *For correspondence: ngilpi@Isuhsc.edu
}

\begin{abstract}
[Abstract] Alcohol use disorder (AUD) is a significant public health and economic burden and is often characterized by repeated bouts of alcohol intoxication and withdrawal. Virtually all organ systems are impacted by chronic alcohol exposure. These effects can be investigated using the rat as a model organism; however, rats typically will not self-administer alcohol to levels necessary to achieve physiological and behavioral aspects of dependence. The protocol described herein can be utilized to induce alcohol dependence in rats by administering alcohol vapor to the homecage for an extended period of time. This method allows the researcher to control the level, duration, and pattern of intoxication, and it reliably induces physiological and behavioral aspects of alcohol dependence, allowing for the study of biology and behavior with relevance for AUD in humans.
\end{abstract}

Keywords: Alcohol dependence, Alcohol use disorder, Vapor, Withdrawal, Intoxication, Blood alcohol level, Rat

[Background] Alcohol use disorder (AUD) affects over 15 million Americans (SAMHSA, 2015), costing the US $\sim 249$ billion annually (Sacks et al., 2015). The progressive development of AUD is characterized by repeated periods of alcohol intoxication and withdrawal, which initiates a series of neuroadaptations and behavioral changes. Individuals with AUD drink excessive quantities of alcohol, partly because alcohol has both positive (i.e., drinking alcohol for its rewarding effects) and negative (i.e., drinking alcohol to remove or avoid aversive feelings) reinforcing effects in those individuals (Koob, 2003). Alcohol-dependent rats withdrawn from alcohol exhibit many of the same behaviors exhibited by alcoholdependent humans, including increases in alcohol drinking (e.g., Roberts et al., 1996), motivation to obtain alcohol (e.g., Walker and Koob, 2007), anxiety-like behavior (e.g., Kallupi et al., 2014) and nociception (e.g., Egli et al., 2012; Avegno et al., 2018). These behaviors suggest that rat models of alcohol dependence are a useful tool for investigating the neurobiological underpinnings of alcohol dependence in humans.

Various experimental methods are available for achieving the high blood alcohol levels (BALs) needed to induce alcohol dependence in rats, including gavage (Majchrowicz, 1975), diet (Lieber and DeCarli, 1982), intraperitoneal injections (Varlinskaya and Spear, 2004), and vapor exposure (Rogers et al., 1979). Vapor exposure allows the experimenter control over the amount, duration, and pattern of alcohol administration. Alcohol is administered passively through vapor delivered into the homecage air supply, decreasing the frequency of handling and/or restraint required for some other alcohol administration 
procedures. The experimenter controls the amount of alcohol administered to each cage, allowing for titration of BALs and achieving reasonably uniform intoxication levels among subjects in a given experiment. Rats exhibit physiological and behavioral signs of alcohol dependence after a few weeks. Behavioral testing can occur during acute withdrawal or protracted abstinence from alcohol vapor; the former allowing for repeated testing (e.g., within-subjects drug dose-response) at the same withdrawal time point on different days. In this protocol, we provide detailed, step-by-step methods updated from previous protocols (Gilpin et al., 2008) for induction of alcohol dependence using chronic intermittent exposure (CIE) to alcohol vapor in rats.

\section{Materials and Reagents}

1. Razor blade (VWR, catalog number: 55411-055)

2. $1.5 \mathrm{ml}$ microtubes (Phenix Research Products, catalog number: $\mathrm{MH}-815$ )

3. Rats (e.g., Wistar rats, 8 weeks of age; Charles River)

4. $95 \%(v / v)$ ethanol (Decon Labs, Koptec, catalog number: V1105)

5. Alcohol oxidase/buffer reagent (Analox Instruments, catalog number: GMRD-113)

6. $100 \%(v / v)$ ethanol (Decon Labs, Koptec, catalog number: V1001G)

7. Liquinox (Alconox, catalog number: 1232)

\section{Equipment}

1. Pre-assembled alcohol vapor inhalation system (La Jolla Alcohol Research, Inc.) or equivalent homemade system

2. High-speed microcentrifuge (Fisher Scientific, AccuSpin Micro 17, catalog number: 13-100-675)

3. Analox AM1 analyzer (Analox Instruments, P-GM7Micro-Stat, catalog number: Analox-AM1)

4. Five-gallon carboy (Fisher Scientific, Thermo Scientific Nalgene Carboy, catalog number: 02960-20A)

5. Power strip with a timer (Century, model: BNC-U1)

6. Animal scale (OHaus, Valor 3000 Xtreme, model: V31XH2)

\section{Procedure}

1. Prepare vapor chamber by ensuring the pre-assembled air and vacuum lines and pressure gauges are securely connected to the air and vacuum lines for the vapor inhalation system (Figure 1).

2. Connect ethanol delivery line to a five-gallon carboy containing $95 \%(v / v)$ ethanol and secure the cap (Figure 1A).

Note: Do not use $100 \%$ ethanol, as it is toxic to animals, and do not use ethanol that is denatured or contains methyl ethyl ketone (MEK), denatonium benzoate, or benzonite. 


\section{biö-protocol

A

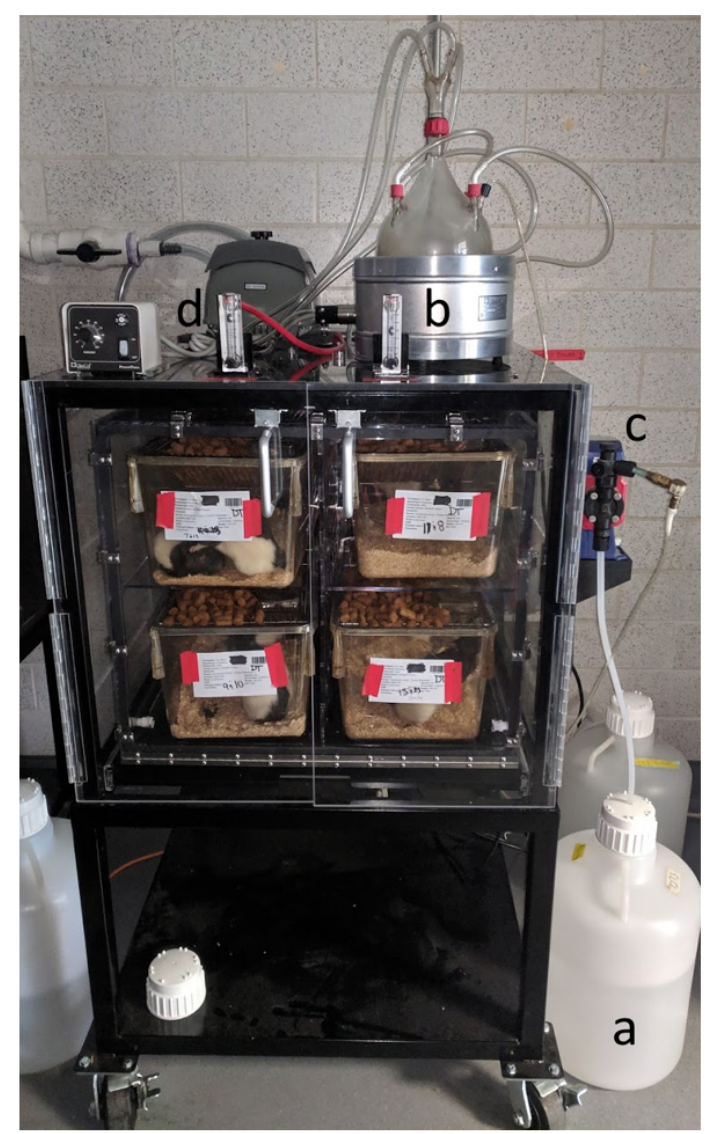

B

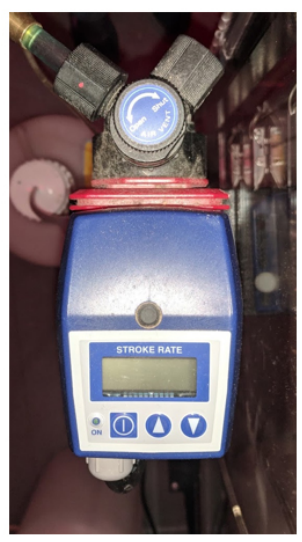

C

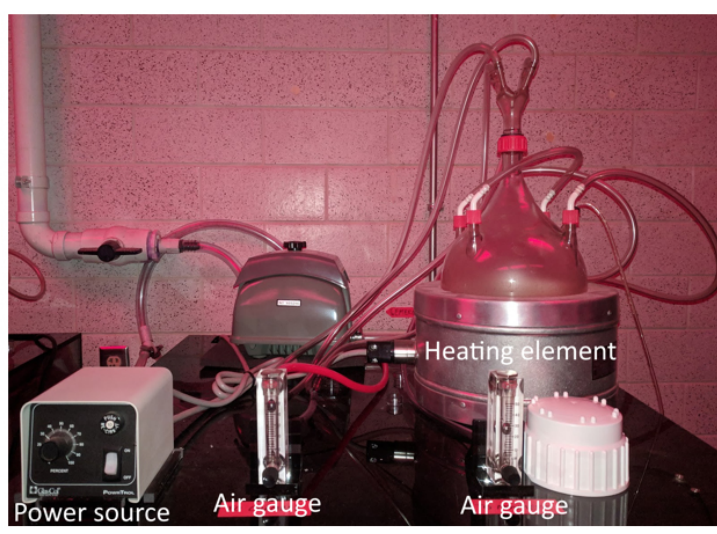

Figure 1. Assembled vapor inhalation system. A. Complete system. $95 \%(v / v)$ ethanol is delivered from a 5-gallon carboy reservoir (a) to the glass flask (b). A heating element is used to generate ethanol vapor, which is delivered to cages housed in the setup via incoming air lines. The temperature of the air delivered to the cages is not appreciably altered by this process. The amount of ethanol delivered through the air lines is controlled by the pump (c), which controls the frequency and volume of ethanol deliveries to the heated flask, where it is evaporated. Air flow is monitored via the air gauge (d). B. Close-up image of the pump. C. Close-up image of heating element, power source for heating element, and air gauge.

3. Adjust pump settings to desired levels, based on animal weight and desired BALs.

Note: These settings can be guided by a general rubric provided by the manufacturer. It is best to start at lower pump settings and gradually increase BALs over the course of the first week, rather than having rats start at a high BAL from session one (see Table 1 for an example). Further considerations for pump settings can be found in the Notes section of this protocol.

4. Set the timer on the power strip to the desired settings. In our experiments, the ethanol pump (plugged into the power strip) is set to ON for $14 \mathrm{~h}$ a day, and OFF $10 \mathrm{~h}$ a day.

Note: Vapor ON/OFF times can differ, but the experimental demands may dictate this schedule. The experimenter should be able to access animals at specific time points in the "vapor ON" and "vapor OFF" phases for measurement of BALs (at least once weekly, more often in the 
beginning of the exposure) and behavioral testing. For our experiments, we often set vapor ON at 6:00 PM and vapor OFF at 8:00 AM, with behavioral tests occurring at approximately 3:00 $P M$. Lights in our vapor room turn OFF at 8:00 AM, which means that most of the alcohol vapor exposure occurs during the light cycle and testing occurs during the dark cycle.

5. Remove lid of the rats' home cage, but keep the wire lid with food and water reservoirs in place. Insert cages into vapor inhalation system and secure the enclosure.

Note: Never turn off incoming air to the vapor inhalation system when animals are inside the vapor inhalation system. If air pressure drops (detectable with the air gauge), remove cages immediately. When combining CIE with behavioral experiments that utilize repeatable procedures, it is ideal to collect baseline behavioral data (e.g., hindpaw withdrawal threshold using a Hargreaves apparatus for nociception, or lever pressing for operant self-administration studies; baseline data should be collected until individual behavior stabilizes to within $20 \%$ variability between sessions), and use those data to assign rats to "vapor" or "air" exposed groups that are counterbalanced for baseline behavioral data. Rats should be weighed before the first exposure to vapor and throughout the CIE procedure (e.g., twice per week).

6. Perform vapor exposure, and monitor BALs. At the vapor OFF time point following the first session in vapor, monitor rats for intoxication by visually assessing somatic symptoms (e.g., gait, respiration, locomotion) while rats are in the homecage (e.g., Nixon and Crews, 2002). Using a razor blade, make a cut at the tip of the tail for blood collection; gently apply pressure to the length of the tail to encourage blood flow, and use a $1.5 \mathrm{ml}$ microtube to collect blood from each animal (volume needed may differ based on the assay being used to measure BALs; for our experiments, we collect $\sim 100 \mu \mathrm{l}$ ). This procedure can be performed with minimal restraint; place the rat on the experimenter's non-dominant arm, encouraging the rat's head to nestle in between the elbow and body, and use non-dominant hand to secure the rat's tail. Place the rat's tail on a sterile surface, and use the dominant hand to nick the tip of the tail. Return rats to homecage when finished collecting blood. Blood should also be collected from air-exposed controls to match for the stress of this procedure.

Note: Microtubes containing heparin or other anticoagulant may be used; these should not interfere with BAL measurement using the Analox system (Step 7). Each time a change is made to alcohol pump settings, blood should be collected to measure BALs at that new setting. This procedure should be repeated until rats achieve the desired BAL range (in our work, typically 150-200 mg/dl). After the target BAL range is achieved, BALs should be measured at least once weekly thereafter to detect and account for metabolic tolerance and/or potential drift in settings. Adjustments will likely be needed as rats develop metabolic tolerance for alcohol following repeated exposures. If combining with behavioral experiments, perform tail bleeds on days when animals are not being tested. Air-exposed controls should be handled and bled on a schedule that is matched to alcohol vapor-exposed rats.

7. Centrifuge blood samples at 9,500 $\times \mathrm{g}$ for $12 \mathrm{~min}$ at room temperature to separate serum from blood. Determine BALs by running serum samples on an Analox AM1 analyzer, using a 
reconstituted alcohol oxidase/buffer reagent, according to the manufacturer's instructions. An ethanol standard, used to calibrate the Analox prior to running serum samples, should be as close as possible to the anticipated range of BALs. In our experiments, we prepare a $100 \mathrm{mg} / \mathrm{dl}$

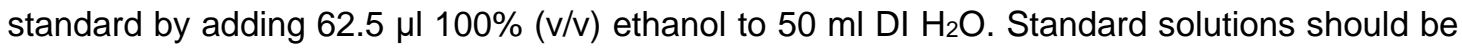
prepared frequently (at least every 1-2 weeks) to ensure accurate concentration.

Note: If animals are extremely impaired (e.g., loss of locomotor activity, loss of muscle tone, no reaction to touch), this likely indicates extremely high BALs (can be confirmed by measuring $B A L s)$. If this exposure level is not the aim of the study, animals should be removed from alcohol vapor overnight to allow BALs to return to zero before the start of the next exposure. Consult with veterinary staff if additional intervention is necessary (e.g., saline administration if rat appears dehydrated; liquefied food if unable to eat solid food; placing on a heating pad if body temperature decreases; pharmacological treatment if severe withdrawal symptoms [e.g., seizures] are present). Adjust pump settings as necessary to reduce BALs. If unable to access an Analox AM1 analyzer, alternate methods for measuring BALs, such as commercially available enzymatic assays and gas chromatography-mass spectrometry, are available. If alternate methods are utilized, adjust the blood collection procedure (e.g., sample storage, centrifuge settings) as necessary.

8. Monitor rats throughout the CIE period. Assess rats daily for general health and well-being by visually observing behavior. Check food and water amounts daily, and refill when necessary. Replace bedding once weekly, on non-test days.

Note: Vapor delivery to the cage generally does not result in wet food or bedding; in the event of condensation buildup, transfer animals to a clean, dry cage, and replace food and bedding. Withdrawal symptom severity can be assessed by the experimenter using desired metrics. While described in greater detail elsewhere (Majchrowicz, 1975; Faingold, 2008), symptoms that manifest during the withdrawal period, as BALs approach 0, can range from mild (e.g., general hyperactivity) to more severe (e.g., convulsive seizures). In the event of seizures, animals may be treated with a benzodiazepine or returned to alcohol vapor to relieve symptoms. It is best to consult with your institution's veterinarian to develop an intervention plan prior to initiating experiments.

A very rough estimation of the rate at which rats typically metabolize ethanol is approximately $25 \mathrm{mg} / \mathrm{dl} / \mathrm{h}$. Tests or tissue collection intended to access the intoxication or withdrawal stages should be scheduled accordingly (i.e., when BALs are peaking or equal to zero). For example, rats being tested for acute withdrawal effects might be maintained in a target range of 150-200 mg/dl and tested or sacrificed at 6-8 $h$ after vapor OFF.

9. Following completion of the experiment, turn the vapor inhalation system off. Clean cages with a mild detergent (e.g., Liquinox), and allow all components (cages and air/vacuum hoses) to completely dry. 


\section{Data analysis}

Data analysis will typically differ based on the aims of the experiment and the in vivo and/or ex vivo procedures being used. If $\mathrm{CIE}$ is combined with behavioral testing, rats should be tested until a behavioral phenotype manifests (for example, escalated alcohol drinking and hyperalgesia emerge on the order of weeks; e.g., Somkuwar et al., 2016). The experimental aims will dictate the testing and end-point schedule, both in terms of the total duration of CIE, as well as in terms of the test or sacrifice timepoint relative to "vapor OFF." In our experiments, rats are tested during the acute withdrawal period. Rats should always be exposed, treated and tested in parallel with air-exposed controls, which allows for statistical comparison of these two groups on all variables being tested (e.g., one-way or two-way ANOVA; for example, see Avegno et al., 2018). If CIE is used to prepare tissue for molecular biology or electrophysiology experiments, tissue harvesting often occurs after $\geq 4$ weeks of CIE (with time $=0$ corresponding to the first session in which rats exhibited BALs in the desired range). Correlational analyses can be used to explore the relationship between in vivo and/or ex vivo measures and BALs across animals in the same cohort.

In behavioral experiments, rats may be excluded based on pre-determined criteria specific to the behavioral assay (e.g., failure to reach criteria, or following an outlier test). Furthermore, experimenters should be aware that extreme alcohol over-exposure (i.e., extremely high BALs) can produce neurotoxic effects (e.g., Obernier et al., 2002) and/or permanently alter behavior. The weight of each rat should be monitored throughout the experiment; weight loss of $>10 \%$ may also be justification for removal of a subject from analysis (consult your veterinarian and IACUC), as this may indicate poor health outcome that can influence other variables of interest.

\section{$\underline{\text { Notes }}$}

If desired, air-ethanol concentrations can be monitored with a breathalyzer apparatus. In order to achieve this, one only needs a port with a screw cap that can be removed to allow for air to escape into the breathalyzer. This port can be inserted by manufacturer, or by the experimenter in a selfassembled apparatus. Breathalyzers are available from multiple vendors. Typically, in our lab, we use blood ethanol concentrations (not air-ethanol concentrations) as the guide for setting changes. Sample settings for a machine with 4 rat cages and passive exhaust at the beginning of an experiment are shown in Table 1. Usually, these settings are too low to produce intoxication, which is intentional, and we slowly increase settings over days to produce intoxication. Settings would be different for a machine with more than 4 rat cages, or for animals of different size/age.

Individual differences in BALs within a cohort can occur, due to differences in rat size and ethanol metabolism. In our experience with Long-Evans and Wistar rats, a given cohort will typically maintain BALs within a $50 \mathrm{mg} / \mathrm{dl}$ range of each other. Occasionally, considerable differences are observed, with a given rat exhibiting a BAL $100 \mathrm{mg} / \mathrm{dL}$ out of range of the others. Also, the concentration of alcohol vapor being delivered to each cage may differ slightly in some machines. Strategies to 
mitigate individual differences within a given cohort of rats will be dictated by the type of vapor machine being used. For example, if using a machine that delivers alcohol vapor to each cage using individual and separate lines, the experimenter may re-house animals (i.e., house a low BAL rat with a similarly low BAL cagemate) and/or change air flow to each individual cage. This would not be possible in a machine that delivers alcohol vapor to a single enclosed space where all animals are housed, but that type of machine should be subject to somewhat less inter-animal variability. Incremental increases or decreases in air flow (e.g., by $\sim 3$ p.s.i.) can produce slight decreases or increases in BALs, respectively. This strategy should be considered only when minor changes in individual BALs are desired, and air flow to any single standard size rat cage should never be below 10 p.s.i. More crude and sweeping changes in BALs can and should be achieved by changing alcohol pump settings.

Rat strains may differ in terms of intoxication levels and metabolic tolerance in response to the same alcohol vapor settings (e.g., Gilpin et al., 2008). Age is also a consideration; for experiments intended to focus on adulthood, vapor exposure should begin no earlier than 8 weeks of age. Vapor exposure can occur at earlier timepoints (e.g., during adolescence), but interpretation of those data may be different from adult experimental data because alcohol effects on brain and behavior change across the lifespan (Novier et al., 2015). Experiments using adolescent rats may need to use lower pump settings to achieve target BALs, given their smaller size relative to adults; however, adolescent rats demonstrate an increased metabolism and decreased sensitivity to sedative effects of alcohol (Little et al., 1996). Sex may also be an important consideration, both in terms of body weight (female rats are smaller) and estrous cycle if that is an important consideration for the given experiment. CIE can also be used in different species, including the mouse, although settings and exposure schedule typically differ significantly (e.g., Becker and Hale, 1993; Griffin et al., 2009).

Table 1. Sample initial pump settings and corresponding BALs for a group of 8 male Wistar rats. Note that the amount of ethanol delivered to the heated flask at a given pump setting is different for each vapor system, and that pump settings on a given vapor system are not linear (i.e., incremental increases in pump settings do not necessarily translate to equally incremental increases in the amount of ethanol delivered to the system). This underscores the importance of measuring BALs each time an adjustment to the vapor system is made.

\begin{tabular}{|l|l|l|l|}
\hline Day & 1 & 2 & 3 \\
\hline Air Flow (p.s.i.) & 30 & 30 & 30 \\
\hline Pump Setting & 8 & 9 & 10 \\
\hline $\begin{array}{l}\text { Average BAL } \\
\text { (mg/dl; +/- SEM) }\end{array}$ & $101.3(4.6)$ & $104.6(17.2)$ & $154.8(22.9)$ \\
\hline
\end{tabular}




\section{Acknowledgments}

This protocol is supported by National Institute of Health (NIH) grants R01 AA023305 (NWG), R01 AA026531 (NWG), F32 AA025831 (EMA), and V.A. grant 101 BX003451 (NWG). The protocol outlined herein is adapted from Gilpin et al., 2008.

\section{Competing interests}

NWG owns shares in Glauser Life Sciences, Inc., a start-up company with interest in development of therapeutics for treatment of mental illness (no direct link to the current work). EMA declares no competing financial interests.

\section{Ethics}

All procedures were approved by the Institutional Animal Care and Use Committee of the Louisiana State University Health Sciences Center, and were in accordance with the National Institute of Health guidelines.

\section{References}

1. Avegno, E. M., Lobell, T. D., Itoga, C. A., Baynes, B. B., Whitaker, A. M., Weera, M. M., Edwards, S., Middleton, J. W. and Gilpin, N. W. (2018). Central amygdala circuits mediate hyperalgesia in alcohol-dependent rats. J Neurosci 38(36): 7761-7773.

2. Becker, H. C. and Hale, R. L. (1993). Repeated episodes of ethanol withdrawal potentiate the severity of subsequent withdrawal seizures: an animal model of alcohol withdrawal "kindling". Alcohol Clin Exp Res 17(1): 94-98.

3. Egli, M., Koob, G. F. and Edwards, S. (2012). Alcohol dependence as a chronic pain disorder. Neurosci Biobehav Rev 36(10): 2179-2192.

4. Faingold, C. L. (2008) The Majchrowicz binge alcohol protocol: an intubation technique to study alcohol dependence in rats. Curr Protoc Neurosci Chapter 9: Unit 9.28.

5. Gilpin, N. W., Richardson, H. N., Cole, M. and Koob, G. F. (2008). Vapor inhalation of alcohol in rats. Curr Protoc Neurosci Chapter 9: Unit 9.29.

6. Griffin, W. C., 3rd, Lopez, M. F., Yanke, A. B., Middaugh, L. D. and Becker, H. C. (2009). Repeated cycles of chronic intermittent ethanol exposure in mice increases voluntary ethanol drinking and ethanol concentrations in the nucleus accumbens. Psychopharmacology (Berl) 201(4): 569-580.

7. Kallupi, M., Vendruscolo, L. F., Carmichael, C. Y., George, O., Koob, G. F. and Gilpin, N. W. (2014). Neuropeptide YY(2)R blockade in the central amygdala reduces anxiety-like behavior but not alcohol drinking in alcohol-dependent rats. Addict Biol 19(5): 755-757. 
8. Koob, G. F. (2003). Alcoholism: allostasis and beyond. Alcohol Clin Exp Res 27(2): 232-243.

9. Lieber, C. S. and DeCarli, L. M. (1982). The feeding of alcohol in liquid diets: two decades of applications and 1982 update. Alcohol Clin Exp Res 6(4): 523-531.

10. Little, P. J., Kuhn, C. M., Wilson, W. A. and Swartzwelder, H. S. (1996). Differential effects of ethanol in adolescent and adult rats. Alcohol Clin Exp Res 20(8): 1346-1351.

11. Majchrowicz, E. (1975). Induction of physical dependence upon ethanol and the associated behavioral changes in rats. Psychopharmacologia 43(3):245-254.

12. Nixon, K. and Crews, F. T. (2002). Binge ethanol exposure decreases neurogenesis in adult rat hippocampus. J Neurochem 83(5): 1087-1093.

13. Novier, A., Diaz-Granados, J. L. and Matthews, D. B. (2015). Alcohol use across the lifespan: An analysis of adolescent and aged rodents and humans. Pharmacol Biochem Behav 133: 6582.

14. Obernier, J. A., Bouldin, T. W. and Crews, F. T. (2002). Binge ethanol exposure in adult rats causes necrotic cell death. Alcohol Clin Exp Res 26(4): 547-557.

15. Roberts, A. J., Cole, M. and Koob, G. F. (1996). Intra-amygdala muscimol decreases operant ethanol self-administration in dependent rats. Alcohol Clin Exp Res 20(7): 1289-1298.

16. Rogers, J., Wiener, S. G. and Bloom, F. E. (1979). Long-term ethanol administration methods for rats: advantages of inhalation over intubation or liquid diets. Behav Neural Biol 27(4): 466486.

17. Sacks, J. J., Gonzales, K. R., Bouchery, E. E., Tomedi, L. E. and Brewer, R. D. (2015). 2010 national and state costs of excessive alcohol consumption. Am J Prev Med 49(5): e73-e79.

18. SAMHSA. (2015). Substance Abuse and Mental Health Services Administration (SAMHSA). 2015 National Survey on Drug Use and Health (NSDUH). Table 5.6A-Substance Use Disorder in Past Year among Persons Aged 18 or Older, by Demographic Characteristics: Numbers in Thousands, 2014 and 2015.2 Available at https://www.samhsa.gov/data/sites/default/files/NSDUH-DetTabs-2015/NSDUH-DetTabs2015/NSDUH-DetTabs-2015.htm\#tab5-6a.

19. Somkuwar, S. S., Fannon, M. J., Staples, M. C., Zamora-Martinez, E. R., Navarro, A. I., Kim, A., Quigley, J. A., Edwards, S. and Mandyam, C. D. (2016). Alcohol dependence-induced regulation of the proliferation and survival of adult brain progenitors is associated with altered BDNF-TrkB signaling. Brain Struct Funct 221(9): 4319-4335.

20. Varlinskaya, E. I. and Spear, L. P. (2004). Acute ethanol withdrawal (hangover) and social behavior in adolescent and adult male and female Spraque-Dawley rats. Alcohol Clin Exp Res 28(1): 40-50.

21. Walker, B. M. and Koob, G. F. (2007). The gamma-aminobutyric acid-B receptor agonist baclofen attenuates responding for ethanol in ethanol-dependent rats. Alcohol Clin Exp Res 31(1): 11-18. 